\title{
A Study on Approximate and Fine Adjustments by Hand Motion in an Immersive Environment
}

\author{
Noritaka Osawa ${ }^{\dagger}$ and XIangshi ReN ${ }^{\dagger \dagger}$
}

\begin{abstract}
Immersive virtual reality (VR) has long been considered an excellent environment in which to manipulate $3 \mathrm{D}$ virtual objects. However currently used immersive VR user interfaces have limitations. For example, while direct manipulation by hand is easy to understand and to use for approximate positioning, direct manipulation by hand is not suitable for making fine adjustments to virtual objects in an immersive environment because it is difficult to hold an unsupported hand in midair and then to release an object at a fixed point. We therefore propose a method that combines direct 3D manipulation by hand with a virtual 3D gearbox widget that we recently designed. Using this method, hand manipulation is used first to move virtual objects and place them in an approximate position, and then the widget is used to move them into a precise position. The experimental evaluation showed that this combination of direct manipulation by hand and the proposed gearbox is the best of five tested methods in terms of completion ratio of task and subjective preference.
\end{abstract}

\section{Introduction}

In comparison with user interfaces on desktop VR environments using mice or just large format screen systems without manipulation of virtual objects, the user interface in immersive virtual environments still remains challenging. For instance, we believe that interaction techniques using hand should be investigated to achieve good and intuitive interfaces in immersive virtual environments. This paper focuses on this issue.

An immersive virtual environment could offer a 3D content creator a familiar, simpler, and very efficient way to content production. For example, changing the position of virtual objects in an immersive space is easy by using direct hand manipulation, that is, direct manipulation by hand. Such direct manipulation is similar to the manipulation of actual objects in real life, so less specialized knowledge and fewer technical skills are required from the creator. Therefore, once a method for fine-tuning adjustments by hand is provided, the method will demand even fewer technical skills and less specialized knowledge of, for example, 2D GUI tools. Thus, 3D content creation could become within the reach of novice or casual users as well as professionals by using an immersive virtual environment.

Several prototype tools and frameworks have

$\dagger$ National Institute of Multimedia Education

$\dagger \dagger$ Kochi University of Technology been reported 2 (4),8) 10),17) 19) that enable users to manipulate objects in a virtual environment. With these tools and frameworks, objects are manipulated with 3D pointing devices such as the cubic mouse ${ }^{6)}$. We think that physical 3D pointing devices may restrict freedom of body movement in an immersive virtual environment, and that this restriction should be avoided. Direct manipulation by hand is easy to understand and to use for approximate positioning, however it is not considered suitable for making fine adjustments to virtual objects in an immersive environment because it is difficult to hold the hand still in midair without support and to release an object at a fixed point.

Another problem of direct manipulation by hand is the unreliability of task completion for some people. Some people can finish a task, but some people cannot finish a task within a reasonable or fairly long period of time. They often get fatigued of manipulation and cannot complete the task. All people should be able to complete a task within a reasonable period by using a method. Otherwise, the method is useless for some people, that is, it is a method for professionals but not for novice or casual users.

Although inputting numerical values for finetuning is possible, doing so is difficult in an immersive environment. Another possibility is inputting numbers by using the up/down keys, but this is tedious when inputting numbers in a large range. Even if the input device were not a problem, understanding which numbers 
to input would still be difficult for novices or non-professionals.

Consequently, we developed a new virtual 3D gearbox widget (described briefly in Section 2.4.2) with multiple interconnected gears that enable one to make both large and fine adjustments to values ${ }^{13)}$. The virtual $3 \mathrm{D}$ gearbox widget is implemented as a widget in the it $3 d$ class library ${ }^{12)}$, which is a Java class library for $3 \mathrm{D}$ applications. The programmer can specify the gear ratios of interconnected gears, and the appearance of the gears matches the specified gear ratio. Familiarity with gears in the real world means that users can easily understand how the gear ratio changes values. Although the virtual 3D gearbox widgets provide an intuitive and precise interface, it is not as suitable as direct manipulation by hand for making approximate adjustments. Direct manipulation by hand is more efficient than virtual 3D gearbox widgets for approximate adjustments ${ }^{14}$ ).

One way to solve this problem would be to use a combination of direct manipulation by hand and gearbox widget, i.e., direct manipulation by hand can be used to place virtual objects in an approximate position, and then the widget can be used to move them precisely. This must be done in a way that maintains usability, otherwise the combination will be less usable than widgets or hand manipulation.

Some researches have been done on 3D interaction techniques such as the silk cursor $^{22)}$, body-relative interaction ${ }^{11)}$, go-go interaction ${ }^{16)}$, ray-casting interaction ${ }^{3)}$, the image plane interaction ${ }^{15)}$, scaled manipulation $^{5)}$, and IntenSelect ${ }^{7)}$. These use a handheld device such as a stylus to manipulate a virtual object but they use neither virtual gearbox widgets nor direct manipulation by hand. There has been little previous research directly in the area of gearbox widget in fine adjustments. It is possible to apply the scaled manipulation ${ }^{5)}$ to manipulation by hand and the scaled manipulation that uses speed-dependent adjustments will improve the efficiency within a limit of accuracy. However, some people could not complete a task of high accuracy even if some other people could. We need a reliable method for a task of high accuracy.

Research on the virtual gearbox widgets ${ }^{13}$ ) has demonstrated the effectiveness of the gearbox widget in an immersive environment. However, combinations of direct manipulation by hand and the virtual widgets have not been evaluated. A few researches on combinations of direct manipulation by hand and widget for interaction with virtual objects have been presented, e.g., Refs. 3), 20), 21), but they were not used for fine position adjustment. The use of combinations of methods often makes the efficiency lower than the sole use of each method. An appropriate combination of methods should be investigated. The work reported here, which results from a project that attempts to solve problems for manipulations and adjustments in immersive environments, enables efficient and precise adjustments of positions.

\section{Method}

We conducted an experiment to evaluate the usability of the combination of direct manipulation by hand and precise adjustment using a $3 \mathrm{D}$ virtual widget for positioning virtual objects in an immersive environment. The hypothesis was that combination of direct manipulation by hand with manipulation by using gearbox widget would be effective because the hand can move virtual objects and place them in an approximate position, and the gearbox widget can precisely adjust their positions. This would be demonstrated by performance and subjective preference.

\subsection{Experimental Environment}

The experiment was performed in a virtual environment called TEELeX (Tele-Existence Environment for Learning eXploration) ${ }^{1)}$. TEELeX is a kind of surround display system that employs immersive projection technology. It has a large cubic screen, each face of which is 3 meters by 3 meters. Circular polarization is employed to give a stereoscopic view to users. In other words, passive stereo is used. We used one stereoscopic face in the experiment to evaluate the interaction methods in a simple and usual VR environment.

The experiment used a PC-based system. The system runs on a PC workstation (Dell Precision 530 with dual 2-GHz Pentium 4 Xeon processors and a 3DLabs Wildcat II 5110 graphics board supporting dual displays). A Six-DoF (degree of freedom) position tracker (Polhemus Fastrak) and a sensor glove (Virtual Technologies CyberGlove) are used to detect the position and motion of the user's body and hand. The experimental software was developed using the Java programming language, the Java 3D class library, and the it3d library ${ }^{12)}$, which is an interactive toolkit library for 3D ap- 


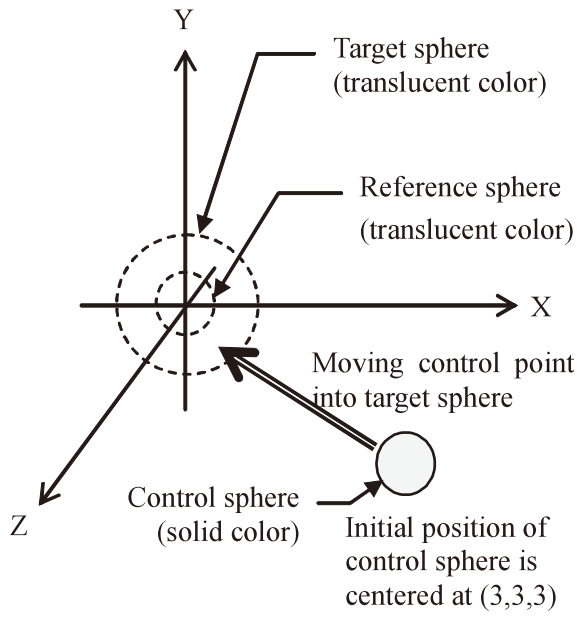

Fig. 1 Experimental task.

plications with artificial reality (AR) technologies.

\subsection{Experimental Task}

The subjects were asked to move a control sphere into a target sphere (translucent) in an immersive virtual environment. Figure 1 illustrates the experimental task. When the control sphere was moved into the target sphere, the control sphere turned blue, indicating to the subject that the control sphere was within the target region. We measured the time taken to move the control sphere from the initial position into the target sphere. Although the subjects were asked to complete the task as quickly as possible, some of them could not complete the task in the specified period in some trials. We had a cut-off time of 2 minutes (120 seconds) because we did not want the subjects to get too tired after one trial. Moreover it is impractical to take more than 2 minutes for one adjustment. A pilot experiment showed that the subjects rarely needed more than 2 minutes to complete the task if they could complete it.

The control sphere was initially centered at $(3,3,3)$ (unit: $\mathrm{cm}$ ) and the target position was at $(0,0,0)$. Those positions are specified by a coordinate system in a position-tracker space, or a physical space. One centimeter is the unit of the position-tracker space. The origin $(0,0,0)$ of the position-tracker space lies at $120 \mathrm{~cm}$ above the center of the floor screen face.

The radius of the control sphere was 1.5 . The target spheres had a radius of 4.5,3.0, 2.0, or 1.7. Orientation was not considered, so a sphere was chosen as the shape of the control and the target in the experiment.
To clarify the depth of the control sphere, an identically sized translucent sphere (reference sphere) was displayed inside the target sphere at $(0,0,0)$. The subjects could understand the depth differences by comparing the size of the control sphere with that of the reference sphere. At the target position, the two spheres were nested. The inner sphere was the reference sphere and the outer sphere was the target sphere.

In order to avoid motion sickness in the experiment, a viewpoint camera was placed at a fixed position $(0,0,40)$ in the position-tracker space and along the $-\mathrm{Z}$ axis direction. In other words, head tracking was not employed in the experiment. The angular field of view of the camera was 90 degrees. Both the horizontal field of view and the vertical field of view were the same because the width and height of the screen face was the same as described in Section 2.1 .

\subsection{Subjects}

Twelve participants ( 5 male, 7 female) took part in the experiment. They were all university students. They were from 19 to 23 years old (mean age $=20.2$ years) with normal vision. They had little or no VR experience and were paid for their participation in the experiment. Each subject performed the task using five methods (described in Section 2.4) in different order.

\subsection{Design}

Regarding the use of a combination of direct manipulation by hand, and the gearbox or slider widgets, there are seven possible interface combinations: direct manipulation by hand alone (referred to as hand), gearbox widget alone (referred to as gear), slider widget alone (referred to as slider), hand+gear, hand+slider, gear+slider, and hand+gear+slider. The use of both gear and slider required too much space, making it impractical. Therefore, we omitted the last two combinations and tested five methods: (1) hand, (2) gear, (3) slider, (4) hand+gear, and (5) hand+slider. In the following subsections, each interaction method will be briefly described. The sole use of (1) hand, (2) gear, or (3) slider is discussed in our previous paper ${ }^{14)}$. Please refer to it for more details.

In all the experiments using each interaction method, the actual scenes were presented to a subject as stereoscopic images. Moreover the widgets and the control sphere were manipu- 


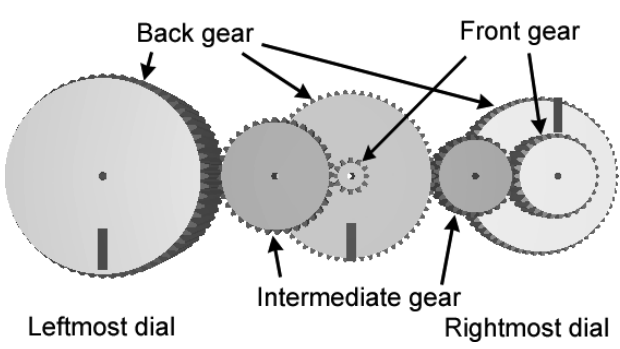

Fig. 2 An example of gearbox widgets.

lated by a hand wearing a sensor glove.

\subsubsection{Direct Manipulation by Hand}

With the direct hand manipulation method, the user pinches the control sphere between his or her thumb and forefinger to hold it, moves the control sphere to the target, and then releases it by opening the hand. Direct manipulation by hand is more similar to real-life actions than the use of a handheld device.

\subsubsection{Gearbox}

Our gearbox widget imitates a physical gearbox virtually and abstractly. Rotating its gears changes a value. In this widget, multiple gears can be interconnected. Familiarity with gears in the real world means that users can easily understand how the gears change the value based on the gear ratio. The appearance of the gears matches the specified gear ratios although the interconnection between gears is not limited to physical structures.

The example of a gearbox widget shown in Fig. 2 has three dials. The back gear (the larger gear) and the front gear (the smaller one), which are concentric, constitute one dial for rotational manipulation. The front gear of the leftmost dial is omitted.

Any two adjacent dials are interconnected with an intermediate gear. The intermediate gear engages with the back gear of the dial at its left and with the front gear of the dial at its right. The intermediate gear is used to maintain the rotational direction of the dials for manipulation. If the intermediate gear is not used, the rotational directions of adjacent dials for increase are different. The intermediate gear does not prevent rotating the dials by hand although this is impossible in the real world. The size of the intermediate gear does not affect the ratios of the adjacent dials.

The leftmost dial is used for large value changes, and the rightmost dial is used for small changes. The value ratios of the dials in Fig. 2 are 10:2:1 for one revolution. The programmer

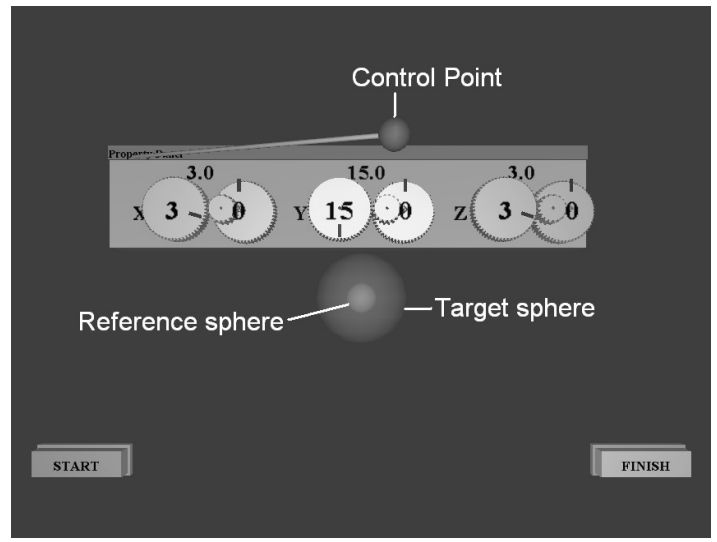

Fig. 3 Monoscopic screenshot of using property panel with gearboxes to perform the task.

can specify the value ratio of a dial for one revolution. The sizes of the back gears are all the same in this example. Thus, the sizes of the front gears are appropriately determined by the gearbox widget based on the specified value ratios. For example, the value ratio of the second dial from the right to the rightmost dial is $2: 1$. Therefore the size ratio of the back gear to the front gear of the rightmost dial is $2: 1$. Similarly, the value ratio of the leftmost dial to the middle dial is $5: 1$.

Experimental settings for the gearbox will be explained in the following. A property panel for the control sphere is used with a virtual 3D gearbox. As shown in Fig. 3, three gearboxes on the panel move the control sphere. The value ratio of the dials for each axis in Fig. 3 is 10:1 for one revolution. A line connected to the control sphere represents the correspondence between the control sphere and the property panel. Selecting and moving the property panel's title bar can move the entire panel. Although Fig. 3 is monoscopic, each subject saw stereoscopic scenes and manipulated virtual gearboxes with his or her hand as stated before.

\subsubsection{Slider}

A property panel with virtual $3 \mathrm{D}$ sliders is shown in Fig. 4. The size of the property panel with sliders is the same as the property panel with gearboxes. The width of each slider track for position control was the same as the width of each dial in the virtual gearbox. Increasing the width of the slider track would make control easier and more precise, but that method would require more space. We conducted the experiment to compare the gearbox method with the slider method on the condition that the prop- 


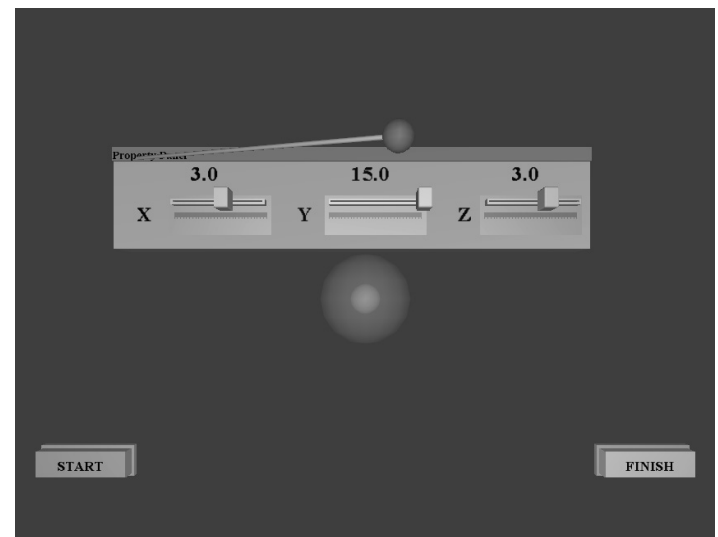

Fig. 4 Monoscopic screenshot of using a property panel with sliders to perform the task.

erty panel sizes were the same.

A vertical arrangement of the sliders increases the width of the slider track but decreases its height. Depending on the size property of the used panel, the height of the slider and the clearance between the sliders are too small to manipulate each slider separately. The small clearance often causes unintended manipulation of adjacent sliders. Therefore we adopted a horizontal arrangement of the sliders in the experiment to remove unintended manipulation from them.

Although it is possible to use multiple interconnected sliders like interconnected gears, the use of multiple sliders causes problems. A slider has an upper limit and a lower limit. When a slider knob reaches its limit, another slider for large change has to be manipulated or the slider knob has to be reset. If another slider for large change is manipulated, the fine adjustments that have been performed become invalid. The reset of the slider knob needs additional manipulations of a button or another widget to change the slider knob, and thus complicates the manipulation for adjustments. Moreover, multiple interconnected sliders are not used in daily life. On the other hand, the gearboxes can use a metaphor from everyday life.

The range of each slider is between -15 and 15. This range is narrower than that used in our previous experiments ${ }^{13), 14)}$ although the range may be too narrow for practical use. The narrow range enables a subject to adjust a value easier than the range used in our pilot experiments.

\subsubsection{Direct Manipulation and Gear- box}

In this method, the position and orientation of the control sphere may be adjusted by the gearbox widgets and direct manipulation by hand. In the experiment, the subjects were not required to use both direct manipulation and gearboxes in one trial. They were free to choose only direct manipulation or only the gearboxes. For example, in a task where the target was large, the subject was expected to use only direct manipulation by hand because that task requires only approximate positioning. The size of gearboxes on the property panels was the same as in the gearbox-only method.

Adjustments by either method can be used in one session, but both methods should not be used simultaneously. Trying to do both simultaneously by using both hands causes a problem in which direct manipulation of the target generally disagrees with manipulation of the widget. For example, let us consider a situation where direct manipulation indicates point $P$ and the widget indicates point $Q$ which is different from $P$. If direct manipulation has precedence over the widget, precise adjustments by manipulating the widget are overridden by approximate adjustments through direct manipulation. This makes the widget useless. Conversely, if the widget takes precedence on direct manipulation, the target moves away from the hand position and the target is released from the hand. Both cases are undesirable, however, we think that direct manipulation should have precedence over the widget because some slips in dialing are tolerable but disagreement between the hand position and the object position are non-intuitive and intolerable.

\subsubsection{Direct Manipulation and Slider}

The method of using both direct manipulation and sliders is similar to the method of using both direct manipulation and gearboxes. The main difference is that the sliders are placed on the property panel to adjust a control sphere. The size of sliders on the panel is the same as in the slider-only method.

\subsection{Procedure}

We used a within-subject design. For each method, the functions of the system and the task were explained to the subjects, and each subject was given practice tasks to learn how to use the sensor glove and the interaction methods. These practice sessions were followed by data collection sessions. The experiment was 
held for two days. The subject performed each task once a day. That is, the subject performed each task twice in the total experiment.

After they finished testing all the methods, the subjects were asked to complete a questionnaire. The question was: "How do you rate the method tested just now? Please rate each item on a scale of 0 to 9 ." In the scale, $0=$ lowest preference, and $9=$ highest preference. The question consisted of six items related to speed, accuracy, ease of use, fatigue, satisfaction, and the desire to use.

\section{Results and Discussion}

\subsection{Incomplete trials}

Figure $\mathbf{5}$ shows the number of incomplete trials for the methods and target sizes. Each subject tried each task twice, and the number of the subjects was 12 . Thus, the number of all trials was 24. Figure 5 shows that the methods of only using sliders or only using direct manipulation by hand resulted in some incomplete trial when the target sizes were small (1.7 or 2), whereas all subjects could complete the task by using the gearbox widgets. The incomplete trial means that the subject did not complete the task within the time limit (120 seconds in this experiment). The difference of the number of the incomplete trials between the hand-only and the hand+gear was significant $(p<0.0001)$ with a two-tailed Fisher's exact test, and the difference between the slider and hand+gear was significant $(p<0.05)$ where the target size was 1.7.

These results showed that the combination of the hand and the gearbox widget is better than hand-only or sliders and thus useful for precise adjustments. The results also showed that the slider, which is often used in GUIs to control coordinates, is unsuitable for fine-tuning in the

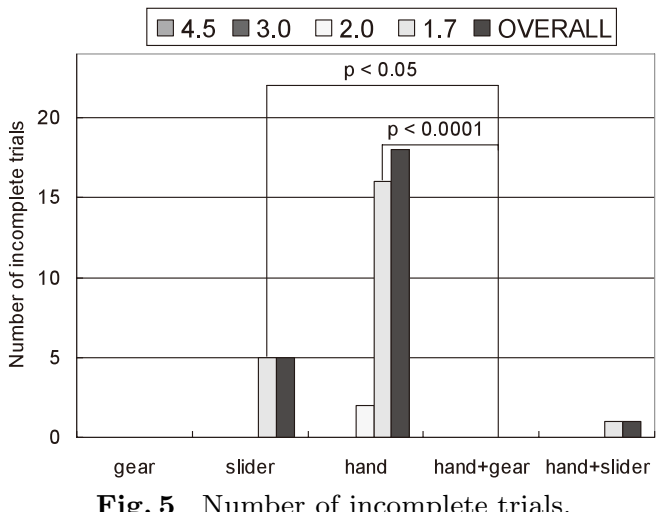

Fig. 5 Number of incomplete trials. immersive environment since it is difficult to move the slider precisely with an unsupported hand.

When the virtual 3D gearbox widgets were not used, some subjects could not complete some tasks. Direct manipulation could not complete the tasks due to measurement errors of a position-tracker and control errors of hand. The virtual 3D gearbox widgets enabled the subjects to complete the tasks within a practical period of time. In real applications, the sole use of an interface method that results in incomplete trials would be impractical. The virtual 3D gearbox widgets and the combinations of direct manipulation and virtual 3D widgets could improve this situation. In other words, they were reliable methods to complete a task within a reasonable period of time.

This experiment used the narrower range $[-15,15]$ of a slider than $[-30,30]$ in the previous experiment. This setting was favorable to the slider but the result showed that the gearbox was superior to the slider for small targets.

The initial position of the control sphere was nearer to the target in this experiment than the previous experiment but this change of the initial position did not influence superiority of the gearbox for small targets.

\subsection{Target size and performance}

This subsection describes the relationships between target size and performance. As mentioned in Section 3.1, some experimental trials in the slider, the hand, and the hand+slider where the target size was 1.7 , and the hand where the target size was 2 were incomplete. Therefore a simple calculation of the mean completion time is impossible in those cases. Hence, we first show the median of trial time ${ }^{2}$. Median of trial time is shown in Fig. 6. In the figure, the trial time of incomplete tasks is shown as 120 seconds.

The average time of the total trials is also shown in Fig. 7. In calculating the average time, completion time of incomplete trials was counted as 120 seconds, which was the lower bound of completion time even if the actual cutoff time was longer. We evaluated the effects that sizes had on the differences between the five methods. There were significant differences

\footnotetext{
F For readers who are not familiar with the notion, the median, is a robust estimate of the center of a sample of data because outliers have little influence on it. Therefore the median is expected to be a robust index of tendency where some tasks are incomplete.
} 


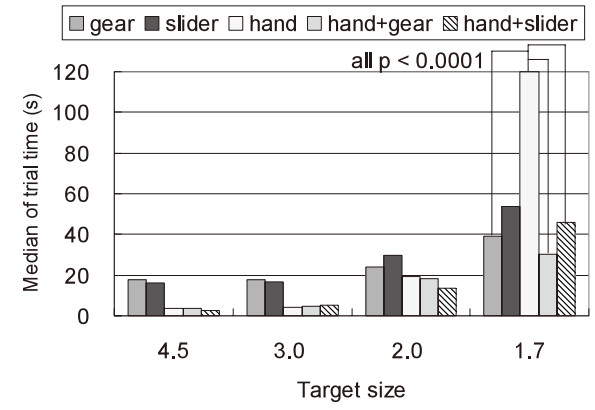

Fig. 6 Median of trial time.

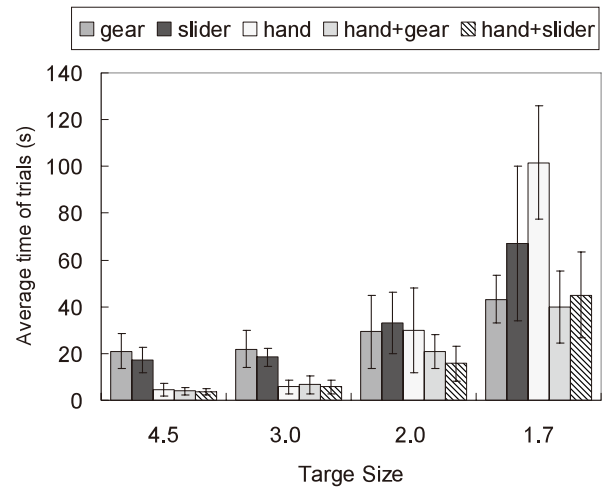

Fig. 7 Average time of trials.

between the five methods for each of the target sizes 2,3 and $4.5, \mathrm{~F}(4,55)=3.97, p<0.05$, 36.12 , and 47.28, respectively, all $p<0.0001$. This means that significant differences in time remained when the target size was varied. The post hoc Tukey HSD test showed that when the target size was 4.5 or 3.0 , hand or hand+gear was better than gear only, $p<0.0001$, and this means that the method of using direct manipulation by hand was superior to the sole use of virtual 3D widgets.

Where the target size was 1.7, there were some incomplete trials when using slider, hand, and hand+slider, thus we used the median test with a two-tailed Fisher's exact test for comparison between the three methods or between each of the three methods and each of the other two methods (i.e., gear, hand+gear). The results showed that the differences between the gear and the hand, between the hand and the hand+gear, and between the hand and the hand+slider were significant where the target size was 1.7 (all $p<0.0001$ ) as shown in Fig. 6 . The result thus shows that when the target size was 1.7 , the hand-only method was worse than other methods. When the target size was

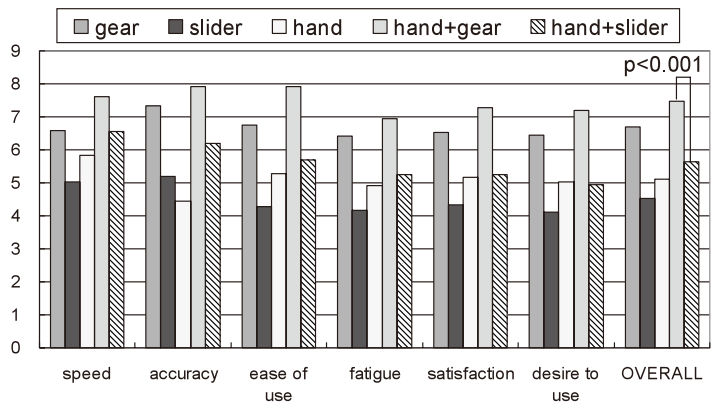

Fig. 8 Subjective preference of the five methods $(0=$ lowest preference, $9=$ highest preference).

1.7, using either the slider method (average $=$ $68.8 \mathrm{~s}$ ) or the hand method (average $=103.2 \mathrm{~s}$ ) took more time to complete the tasks than the gear method (average $=43.3 \mathrm{~s}$ ) or the hand +gear (average $=40.9 \mathrm{~s}$ ) and hand+slider $($ average $=46.1 \mathrm{~s})$ combination methods. However, there was no significant difference between gear and hand+gear at 1.7.

\subsection{Preference}

Figure 8 shows the respective subjective ratings and the overall ratings. The overall ratings were based on the average value of the answers given by the subjects to the six question items. The hand+gear method was most preferred $($ mean $=7.49)$. A significant difference was found in the overall ratings of the five methods, $\mathrm{F}(4,25)=39.09, p<0.0001$.

The hand+gear method was also rated as best for all questions. The second best was the gearbox method for all questions. The worst was the slider method. A significant difference was also found between the hand+gear and hand+slider methods, $\mathrm{F}(1,10)=37.1$, $p<0.001$. We think that this difference resulted from the quantitative differences like the number of incomplete trials.

These results show that the hand+gear method was better than the hand+slider method. The qualitative results also show that the combination had a significant effect on subjective preference. In all of the categories, subjective preferences were significantly rated high when the combination was used (see Fig. 8).

Taking the results of Sections 3.1, 3.2 and 3.3 together, the hand+gear combination method is the best of the five methods for a wide range of accuracy. This range includes low accuracy in which the gear was not the best.

\section{Conclusion}

We have focused on intuitive interfaces in an 
immersive virtual environment and have proposed an interface that combines direct manipulation by hand and gearbox widget adjustment. We then evaluated the hand+gear method through the experiment.

The results of the experiment show that, in terms of manipulation time and subjective evaluation, the hand+gear method was better than other combination methods and better than the individual use of hand, gear, or slider. The quantitative results support the hypothesis that the combination of hand+gear will improve the usability. The hand manipulation helps users move a virtual object directly, approximately and efficiently and the gearbox widget helps users move a virtual object indirectly and precisely. The combination of hand+gear makes it significantly easier for a user to manipulate a virtual object efficiently and precisely.

In summary, our proposed technique efficiently enables users to adjust the position of a virtual object both approximately and precisely. These results give designers a simple way to increase the usability of manipulation in immersive virtual environments. Future work includes investigation on other combinations of input interfaces.

Acknowledgments We would like to thank the anonymous reviewers and the metareviewer for their valuable comments.

This research was partially supported by a Grant-in-Aid for Scientific Research (14380090, 19650251), and by the R\&D support scheme for funding selected IT proposals of the Ministry of Public Management, Home Affairs, Posts and Telecommunications (Japan).

\section{References}

1) Asai, K., Osawa, N. and Sugimoto, Y.: Virtual Environment System on Distance Education, EUROMEDIA '99, pp.242-246 (1999).

2) Barnes, G.: Visual Programming Agents for Virtual Environments, 2000 AAAI Spring Symp., pp.173-177.

3) Bowman, D. and Hodges, L.: An evaluation of techniques for grabbing and manipulating remote objects in immersive virtual environments, Symposium on Interactive 3D Graphics, pp.35-38 (1997).

4) Conner, B., et al.: Three-dimensional widgets, Interactive 3D graphics Symposium 1992, pp.183-188 (1992).

5) Frees, S. and Kessler, G.D.: Precise and Rapid Interaction through Scaled Manipulation in Immersive Virtual Environments, IEEE Vir- tual Reality 2005, pp.99-106.

6) Fröhlich, B. and Plate, J.: The cubic mouse: A new device for three-dimensional input, $\mathrm{CHI}$ 2000, pp.526-531.

7) Haan, G. de, Koutek, M. and Post, F.H.: IntenSelect: Using Dynamic Object Rating for Assisting 3D Object Selection, 9th Int. Workshop on Immersive Projection Technology, 11th Eurographics Workshop on Virtual Environments (IPT/EGVE 2005), pp.201-209 (Oct. 2005).

8) Lee, G.A., Kim, G.J. and Park, C.-M.: Modeling virtual object behavior within virtual environment, ACM Symposium on Virtual Reality Software and Technology 2002, pp.41-48, Hong Kong, China (2002).

9) Holm, R., Stauder, E., Wagner, R., Priglinger, M. and Volkert, J.: A Combined Immersive and Desktop Authoring Tool for Virtual Environments, IEEE Virtual Reality Conference 2002, pp.93-100 (2002).

10) Kitamura, Y., Ogata, S. and Kishino, F.: A manipulation environment of virtual and real objects using a magnetic metaphor, ACM Symposium on Virtual Reality Software and Technology 2002, pp.201-207 (2002).

11) Mine, M., Brooks, F. and Sequin, C.: Moving objects in space: exploiting proprioception in virtual-environment interaction, SIGGRAPH'97, pp.19-26 (1997).

12) Osawa, N., Asai, K. and Saito, F.: An Interactive Toolkit Library for 3D Applications: it3d, Eighth Eurographics Workshop on Virtual Environments (EGVE2002), pp.149-157 (May 2002).

13) Osawa, N. and Ren, X.: Gearbox Widget for Fine Adjustments by Hand Motion, IPT/EGVE 2003 Workshop, pp.313-314 (May 2003).

14) Osawa, N. and Ren, X.: Virtual 3D gearbox widget technique for precise adjustment by hand motion in immersive VR, IEICE Trans. Inf. Syst., Vol.E87-D, No.10, pp.24082414 (2004).

15) Pierce, J., et al.: Image plane interaction techniques, in 3D immersive environments, Symposium on Interactive $3 D$ Graphics 1997, pp.3943 (1997).

16) Poupyrev, I., Billinghurst, M., Weghorst, S. and Ichikawa, T.: Go-Go Interaction Technique: Non-Linear Mapping for Direct Manipulation in VR, UIST'96, pp.79-80 (1996).

17) Pinho, M.S., Bowman, D.A. and Carla, M.D.S.: Cooperative object manipulation in immersive virtual environments: Framework and techniques, ACM Symposium on Virtual Reality Software and Technology 2002, pp.171- 
178 (2002).

18) Thalmann, D.: Using Virtual Reality Techniques in the Animation Process, Virtual Reality Systems, Earnshaw, R., Gigante, M. and Jones, H. (Eds.), Academic Press, pp.143-159.

19) Thalmann, N.M. and Thalmann, D.: 3-D Devices and Virtual Reality in Human Animation, 2nd Eurographics Workshop on Animation and Simulation, Vienna (1991).

20) Ware, C. and Rose, J.: Rotating virtual objects with real handles, ACM Transaction on Computer-Human Interaction, Vol.6, No.2, pp.162-180 (1999).

21) Zhai, S., Milgram, P. and Buxton, W.: The influence of muscle groups on performance of multiple degree-of-freedom input, $\mathrm{CHI}^{\prime} 96$, pp.308-315 (1996).

22) Zhai, S., et al.: The Partial Occlusion Effect: Utilizing Semi-transparency in 3D Human Computer Interaction, ACM Transactions on Computer Human Interaction, Vol.3, No.3, pp.254-284 (1996).

(Received December 25, 2006)

(Accepted August 9, 2007)

(Released November 7, 2007)

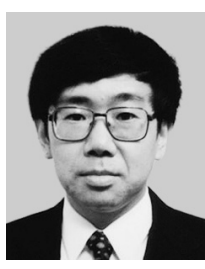

Noritaka Osawa received his B.S., M.S. and Ph.D. degrees in information science from the University of Tokyo in 1983, 1985 and 1988 respectively. After working for a software company and the University of Electro-Communications, he has been at the National Institute of Multimedia Education since 1998. He has also been jointly appointed at the Graduate University for Advanced Studies since 2001. He now works for them as a professor. His current research interests include human-computer interfaces using virtual reality technologies, information visualization and system software.

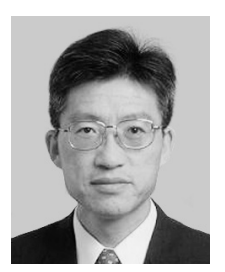

Xiangshi Ren is an Associate Professor in Department of Information Systems Engineering at Kochi University of Technology. He received a B.E. degree in electrical and communication engineering, and M.E. and Ph.D. degrees in information and communication engineering from Tokyo Denki University, Japan, in 1991, 1993, and 1996, respectively. He was an instructor in Department of Information and Communication Engineering at Tokyo Denki University from 1996 to 1999. After working for Tokyo Denki University, he has been at Kochi University of Technology since 2000. His research interests include all aspects of human-computer interaction, in particular, multimodal interactions, and user interface design and evaluation. He is a senior member of the IEEE, a member of the ACM, the IPSJ, the IEICE, and the Human Interface Society. 